\title{
Plasma soluble CD36, carotid intima-media thickness and cognitive function in patients with type 2 diabetes
}

Xiaozhen Jiang ${ }^{1}$, Xiaohui Zhao ${ }^{2}$, Ruihua Chen ${ }^{1}$, Quan Jiang ${ }^{3}$, Bin Zhou ${ }^{1}$

${ }^{1}$ Department of Endocrinology, Shanghai Pudong New Area People's Hospital, Shanghai, China

${ }^{2}$ Department of Neurology, Shanghai Pudong New Area People's Hospital, Shanghai, China

${ }^{3}$ Department of Ultrasonography, Shanghai Pudong New Area People's Hospital, Shanghai, China

Submitted: 31 July 2015

Accepted: 7 November 2015

Arch Med Sci 2017; 13, 5: 1031-1039

DOI: 10.5114/aoms.2016.60821

Copyright @ 2016 Termedia \& Banach

\author{
Corresponding author: \\ Ruihua Chen \\ Department of Endocrinology \\ Shanghai Pudong \\ New Area People's Hospital \\ No. 490 South \\ Chuanhuan Road \\ Pudong, Shanghai, China \\ Phone: +86 18917901822 \\ E-mail: chenruihua55@126. \\ com
}

\begin{abstract}
Introduction: Diabetes and atherosclerosis are both risk factors of cognitive deficits. Scavenger receptor CD36 is associated with increasing risk of diabetes and atherosclerosis, and may play a role in cognitive deficits. The aim of this study is to determine the correlations of plasma soluble CD36 concentrations with carotid intima-media thickness (IMT) and cognitive function in patients with type 2 diabetes.

Material and methods: We determined the levels of soluble CD36 (sCD36), blood lipids, fasting blood glucose, glycosylated hemoglobin, carotid atherosclerosis as IMT, cognitive function by the Montreal Cognitive Assessment (MoCA) scoring system, and other clinical characteristics in 357 patients with type 2 diabetes.

Results: Diabetic patients with the lowest quartile of IMT (Q1) had lower SCD36 concentrations (ANOVA, $p_{\text {trend }}<0.05$ ) and higher MoCA scores than upper ones (Q2-Q4) ( $\left.p_{\text {trend }}<0.05\right)$, and those with the highest quartile of SCD36(Q4) had higher FBG, LDL-C and carotid IMT than lower ones (Q1-Q3) $\left(p_{\text {trend }}<0.05\right.$ for all). Plasma $\log _{10}(\mathrm{sCD} 36)$ was significantly correlated with carotid IMT $(r=0.202, p<0.001)$ after adjustment for age, gender, and education level. Carotid IMT was significantly associated with MoCA scores $(r=0.284, p<0.001)$ after adjustment for, age, gender, education level, duration of DM and hypertension. There were no correlations between SCD36 and MoCA scores $(r=-0.038, p=0.470)$.

Conclusions: Our study shows that SCD36 is associated with carotid IMT, and carotid IMT is inversely correlated with cognitive function in type 2 diabetic patients. Nevertheless, no cross-sectional association between sCD36 and MoCA scores was detected in this study.
\end{abstract}

Key words: diabetes mellitus type 2, soluble CD36, atherosclerosis, carotid intima-media thickness, Montreal Cognitive Assessment (MoCA).

\section{Introduction}

Diabetes is becoming a major health problem all over the world. Cardiovascular disease (CVD) risk is 2- to 8-fold higher in the diabetic population than it is in non-diabetic individuals of a similar age, sex and ethnicity [1, 2]. Additionally, there is growing evidence that chronic hyperglycemia has various negative effects on the central and peripheral 
nervous system $[3,4]$. Several studies have indicated that diabetes can be a risk factor for the development of mild cognitive impairment $(\mathrm{MCl})$ and dementia [5-7]. Epidemiological and basic science evidence suggest a possible shared pathophys iology between type 2 diabetes mellitus (T2DM) and Alzheimer's disease (AD) [8]. A diagnosis of diabetes increased the odds of cognitive decline 1.2- to 1.7-fold, and the odds of future dementia 1.6-fold [9]. Thus, cognitive dysfunction has been suggested to be one of the chronic complications of diabetes by many investigators.

Besides diabetes, atherosclerosis is also a risk factor of cognitive deficits. Links between atherosclerosis and cognitive impairment in people with [10] and without diabetes [11] are increasingly recognized. Many vascular risk factors, including increasing carotid intima-media thickness (carotid IMT) [12], low ankle brachial index (ABI) [13], hypercholesterolemia [14] and hypertension [15], have been implicated in the development of cognitive decline and dementia.

Scavenger receptor CD36 is a transmembrane glycoprotein expressed in a variety of tissues which has been shown to be involved in angiogenesis, inflammation, lipid metabolism, and atherosclerosis [16-18]. Recently, Park et al. [19] suggested that CD36 may promote vascular amyloid deposition and cerebrovascular damage, leading to neurovascular dysfunction and cognitive impairment. Soluble CD36 (sCD36) in plasma was proposed to reflect tissue CD36 expression and may thus be a potential marker associated with atherosclerosis [20]. A growing body of evidence suggests that elevated SCD36 is associated with carotid atherosclerosis [21], insulin resistance and higher type 2 diabetes risk [22].

Although CD36 may play a role in cognitive impairment, and SCD36 was considered to be correlated with carotid atherosclerosis, little is known about the correlations among plasma soluble CD36 concentrations, carotid IMT and cognitive function in patients with type 2 diabetes. In the present study we aimed to determine the individual and combined associations of sCD36 and carotid and cognitive function of patients with type 2 diabetes in Shanghai, China.

\section{Material and methods}

\section{Patients}

Data collection took place from September $1^{\text {st }}$, 2011 to May $31^{\text {st }}, 2012$. Patients with type 2 diabetes (diagnosed by 1999 World Health Organization criteria), age between 33 and 78, were consecutively enrolled from the outpatient department of our hospital in Shanghai. The Montreal Cognitive Assessment (MoCA) scoring system was used to identify the cognitive function of all the patients.

The exclusion criteria were as follows: (1) diabetic ketoacidosis or other acute diabetic complications in the last 3 months, severe heart failure, chronic renal failure, lung disease, or a history of infection in the central nervous system, stroke, cerebral hemorrhage or other clinical evidence of central nervous damage; (2) history of auditory disorders and psychological disturbances, which might interfere with the MoCA test; (3) history of chronic disease which could cause dementia (including Alzheimer syndrome, Parkinson syndrome, vascular dementia, Huntington's disease, hydrocephalus, brain neoplasm, epilepsy, multiple sclerosis, chronic subdural hematoma, severe head trauma, abnormal brain structure, schizophrenia, etc.); (4) history of alcohol abuse or drug abuse; (5) history of depression and taking antidepressants within 6 months before recruitment; (6) use of possible or known drugs associated with cognitive impairment in the previous month (e.g.: steroids, antiepileptic drug, sedative-hypnotic drugs, anesthetics, etc.).

The study was carried out in accordance with the Declaration of Helsinki (2000) of the World Medical Association and approved by the local medical ethics committee. Written informed consent was obtained from all participants.

\section{Anthropometric measurements}

All the subjects underwent an interview including age, sex, education level, history of smoking, history of drinking, history of hypertension and duration of DM. Data of waist circumference, body mass index (BMI), and blood pressure were collected. Hypertension was defined as systolic (SBP) or diastolic blood pressure (DBP) $\geq 140 \mathrm{~mm} \mathrm{Hg}$, or $90 \mathrm{~mm} \mathrm{Hg}$, respectively, or self-reported current use of blood pressure lowering medication. Medication and supplement use data were obtained for use of lipid-lowering medications, antidiabetes medications, antihypertensive medications, and antiplatelet medications through questionnaires and pill bottle reviews.

\section{Laboratory measurements}

Levels of sCD36 were measured by use of the commercially available enzyme-linked immunosorbent assay (ELISA) kits (BlueGene, Shanghai, China). The inter and intra-assay coefficient of variation (CV) was $15 \%$ and $8 \%$. Fasting blood glucose (FBG), triglyceride (TG), total cholesterol (TC), low-density lipoprotein cholesterol (LDL-C), and high-density lipoprotein cholesterol (HDL-C) were measured enzymatically on an automatic analyzer (Cobas 8000 C701 C502 auto chemistry analyzer, 
Roche, USA). Glycosylated hemoglobin $\left(\mathrm{HbA}_{1 c}\right)$ was quantified from resolved erythrocytes with high performance liquid chromatography (HLC$723 \mathrm{G} 7$ analyzer, TOSOH kabushiki kaisha, Japan).

\section{Ultrasonography of carotid arteries}

Color Doppler ultrasound (Sequoia scanner, SIEMENS, Germany) was used to image the right and left common carotid arteries (CCA). By using an automated edge detection system, IMT was measured between the lumen intima and media-adventitia interfaces of the far wall of the CCA (the $1 \mathrm{~cm}$ segment proximal to the bifurcation). The mean IMT of this $1 \mathrm{~cm}$ segment was measured and the mean of measurements on both sides was used as the carotid IMT.

\section{Statistical analysis}

All continuous data were tested for normality using the Kolmogorov-Smirnov (KS) test. Because of skewness and kurtosis of the distributions, serum triglyceride and plasma sCD36 concentrations were logarithmically transformed for statistical analyses and then back-transformed to their natural units for presentation in text and tables. One-way ANOVA and Tukey's post hoc analysis were used to analyze the difference of clinical characteristics by quartiles (Q1-Q4) of carotid IMT and SCD36. The $\chi^{2}$ test was used for comparison of categorical variables. Univariate analysis was performed using Pearson correlation analysis or Spearman rank correlation. The factors influencing SCD36, carotid IMT and MoCA score were analyzed using partial correlation analyses. These analyses were performed using the Statistical Package for the Social Sciences (SPSS Version 19.0, IBM). Results are expressed as mean \pm SD or median (25-75\%). A $p$-value of less than 0.05 was considered to be significant.

\section{Results}

The demographic, anthropometric, and clinical characteristics of the study population are presented in Table I. On average, the 357 adults included in this analysis were aged $57.1 \pm 9.31$ (range: $33-78$ ) years, 35\% were men, and the mean body mass index was $25.31 \pm 3.28 \mathrm{~kg} / \mathrm{m}^{2}$. Mean duration of diabetes were $6.03 \pm 3.56$ years. Mean education duration was $8.18 \pm 3.06$ years. One hundred and eighty-seven (52.4\%) patients had a history of hypertension. Current smokers numbered 60 (16.8\%) and habitual alcohol drinkers 36 (10.1\%). The mean MoCA score was 23.10 \pm 3.30 .

Table II presents the demographic, anthropometric, and clinical predictors of carotid IMT. Patients with the highest IMT were older, had less education, longer duration of diabetes, had higher proportion of hypertension, waist circumference, SBP, DBP, TC, TG, HDL-C, LDL-C, SCD36, and lower MoCA scores (ANOVA, $p_{\text {trend }}<0.05$ ) (Figure 1).

Table III presents the demographic, anthropometric, and clinical predictors of plasma SCD36. Patients with the highest SCD36 had higher FBG, LDL-C and carotid IMT (ANOVA, $p_{\text {trend }}<0.05$ ) (Figure 2).

Univariate analysis revealed significant correlations between plasma sCD36 and age, sex, edu-

Table I. Descriptive characteristics of the $357 \mathrm{pa}$ tients with type 2 diabetes

\begin{tabular}{|c|c|}
\hline Characteristic & Value \\
\hline Age, mean \pm SD [years] & $57.17 \pm 9.31$ \\
\hline Men, $n(\%)$ & $125(35.0)$ \\
\hline Education, mean \pm SD [years] & $8.18 \pm 3.06$ \\
\hline Duration of DM, mean \pm SD [years] & $6.03 \pm 3.56$ \\
\hline $\mathrm{BMI}$, mean $\pm \mathrm{SD}\left[\mathrm{kg} / \mathrm{m}^{2}\right]$ & $25.31 \pm 3.28$ \\
\hline Waist circumference, mean $\pm \mathrm{SD}[\mathrm{cm}]$ & $85.73 \pm 9.12$ \\
\hline Habitual alcohol drinker, $n(\%)$ & $36(10.1)$ \\
\hline Current smoker, $n(\%)$ & $60(16.8)$ \\
\hline Hypertension, $n$ (\%) & $187(52.4)$ \\
\hline $\mathrm{SBP}$, mean $\pm \mathrm{SD}[\mathrm{mm} \mathrm{Hg}]$ & $132.22 \pm 16.55$ \\
\hline $\mathrm{DBP}$, mean $\pm \mathrm{SD}[\mathrm{mm} \mathrm{Hg}]$ & $83.37 \pm 9.30$ \\
\hline $\mathrm{FBG}$, mean $\pm \mathrm{SD}[\mathrm{mmol} / \mathrm{I}]$ & $9.76 \pm 2.66$ \\
\hline $\mathrm{HbA}_{1 \mathrm{c}}$, mean $\pm \mathrm{SD}(\%)$ & $7.93 \pm 1.10$ \\
\hline $\mathrm{TC}$, mean $\pm \mathrm{SD}[\mathrm{mmol} / \mathrm{l}]$ & $5.19 \pm 1.02$ \\
\hline TG, median (25-75\%) [mmol/l] & $1.33(0.93-1.96)$ \\
\hline $\mathrm{HDL}-\mathrm{C}$, mean $\pm \mathrm{SD}[\mathrm{mmol} / \mathrm{l}]$ & $1.41 \pm 0.36$ \\
\hline $\mathrm{LDL}-\mathrm{C}$, mean $\pm \mathrm{SD}[\mathrm{mmol} / \mathrm{l}]$ & $2.97 \pm 0.78$ \\
\hline MoCA score, mean \pm SD & $23.10 \pm 3.30$ \\
\hline sCD36, median (25-75\%) [ng/ml] & $3.22(2.48-3.96)$ \\
\hline $\mathrm{C}-\mathrm{IMT}$, mean $\pm \mathrm{SD}[\mathrm{mm}]$ & $0.83 \pm 0.18$ \\
\hline Without medication therapy, $n(\%)$ & $41(11.5)$ \\
\hline Use of insulin, $n(\%)$ & $129(36.1)$ \\
\hline Oral antidiabetic medications, $n(\%)$ & $276(77.3)$ \\
\hline Antihypertensive medications, $n(\%)$ & $93(26.1)$ \\
\hline Antiplatelet medications, $n(\%)$ & $17(4.8)$ \\
\hline Lipid-lowering medications, $n$ (\%) & $43(12.0)$ \\
\hline
\end{tabular}

$B M I$ - body mass index, SBP-systolic blood pressure, DBP-diastolic blood pressure, $F B G$ - fasting blood glucose, $H b A_{1 c}$ - glycosylated hemoglobin, TC - total cholesterol, TG - triglycerides, HDL-C - highdensity cholesterol, LDL-C-low-density cholesterol, SCD36-soluble CD36, C-IMT-common carotid IMT. 
Table II. Characteristics of participants according to quartiles of carotid IMT

\begin{tabular}{|c|c|c|c|c|c|c|}
\hline \multirow[t]{2}{*}{ Parameter } & \multicolumn{4}{|c|}{ IMT [mm] } & \multirow[t]{2}{*}{$F / \chi^{2}$} & \multirow[t]{2}{*}{$P_{\text {trend }}$} \\
\hline & $\begin{array}{c}\mathrm{Q} 1 \\
(0.45-0.69) \\
(n=91)\end{array}$ & $\begin{array}{c}\mathrm{Q} 2 \\
(0.70-0.82) \\
(n=89)\end{array}$ & $\begin{array}{c}\text { Q3 } \\
(0.83-0.98) \\
(n=87)\end{array}$ & $\begin{array}{c}\text { Q4 } \\
(0.99-1.25) \\
(n=90)\end{array}$ & & \\
\hline Age, mean $\pm S D$ [years] & $49.68 \pm 6.86$ & $56.76 \pm 8.20^{\mathrm{a}}$ & $59.63 \pm 8.11^{\mathrm{bc}}$ & $62.77 \pm 8.62^{\mathrm{abc}}$ & 126.08 & $<0.001$ \\
\hline Men, $n(\%)$ & $26(28.6)$ & $27(30.3)$ & $34(39.1)$ & $38(42.2)$ & 5.203 & 0.158 \\
\hline Education, mean \pm SD [years] & $9.26 \pm 2.21$ & $8.67 \pm 3.31$ & $7.77 \pm 3.05^{\mathrm{ab}}$ & $6.98 \pm 3.11^{\mathrm{ab}}$ & 31.753 & $<0.001$ \\
\hline $\begin{array}{l}\text { Duration of DM, mean } \pm \text { SD } \\
\text { [years] }\end{array}$ & $5.44 \pm 3.10$ & $6.14 \pm 3.60$ & $5.93 \pm 3.80$ & $6.60 \pm 3.65^{\mathrm{a}}$ & 3.874 & 0.050 \\
\hline BMI, mean $\pm \mathrm{SD}\left[\mathrm{kg} / \mathrm{m}^{2}\right]$ & $24.20 \pm 3.07$ & $24.93 \pm 2.95$ & $25.96 \pm 3.45^{\mathrm{ab}}$ & $26.19 \pm 3.27^{\mathrm{ab}}$ & 21.797 & $<0.001$ \\
\hline $\begin{array}{l}\text { Waist circumference, } \\
\text { mean } \pm S D[\mathrm{~cm}]\end{array}$ & $81.12 \pm 8.94$ & $85.41 \pm 8.78^{a}$ & $87.40 \pm 8.55^{a}$ & $89.08 \pm 8.29^{\mathrm{ab}}$ & 40.347 & $<0.001$ \\
\hline Habitual alcohol drinker, $n$ (\%) & $10(11.0)$ & $10(11.2)$ & $8(9.3)$ & $8(9.0)$ & 0.385 & 0.943 \\
\hline Current smoker, $n(\%)$ & $12(13.2)$ & $17(19.1)$ & $13(21.7)$ & $18(20.2)$ & 0.953 & 0.329 \\
\hline Hypertension, $n$ (\%) & $22(24.2)$ & $47(52.8)^{\mathrm{a}}$ & $55(63.2)^{a}$ & $63(70.0)^{\mathrm{ab}}$ & 44.327 & $<0.001$ \\
\hline SBP, mean $\pm \mathrm{SD}[\mathrm{mm} \mathrm{Hg}]$ & $\begin{array}{l}125.88 \\
\pm 16.44 \\
\end{array}$ & $\begin{array}{c}131.91 \\
\pm 15.93^{\mathrm{a}} \\
\end{array}$ & $\begin{array}{r}134.43 \\
\pm 15.41^{\text {a }} \\
\end{array}$ & $\begin{array}{r}136.81 \\
\pm 16.58^{\mathrm{ab}} \\
\end{array}$ & 21.70 & $<0.001$ \\
\hline DBP, mean \pm SD $[\mathrm{mm} \mathrm{Hg}]$ & $80.82 \pm 9.70$ & $83.54 \pm 8.99^{a}$ & $84.76 \pm 9.24^{a}$ & $84.44 \pm 8.88^{a}$ & 7.765 & 0.006 \\
\hline $\mathrm{FBG}$, mean $\pm \mathrm{SD}[\mathrm{mmol} / \mathrm{l}]$ & $9.39 \pm 2.47$ & $9.61 \pm 2.55$ & $10.16 \pm 2.66$ & $9.90 \pm 2.94$ & 2.737 & 0.099 \\
\hline $\mathrm{HbA}_{1 \mathrm{c}}$, mean $\pm \mathrm{SD}(\%)$ & $7.74 \pm 1.00$ & $7.93 \pm 1.18$ & $8.08 \pm 1.15^{\mathrm{a}}$ & $7.96 \pm 1.07$ & 2.357 & 0.126 \\
\hline $\mathrm{TC}$, mean $\pm \mathrm{SD}[\mathrm{mmol} / \mathrm{l}]$ & $4.85 \pm 1.03$ & $5.23 \pm 1.00^{\mathrm{a}}$ & $5.27 \pm 0.98^{a}$ & $5.37 \pm 1.00^{\mathrm{a}}$ & 10.759 & 0.001 \\
\hline $\begin{array}{l}\text { TG, median }(25-75 \%) \\
{[\mathrm{mmol} / \mathrm{l}]^{+}}\end{array}$ & $\begin{array}{c}1.08 \\
(0.85-1.50)\end{array}$ & $\begin{array}{c}1.31 \\
(0.93-2.13)^{\mathrm{a}} \\
\end{array}$ & $\begin{array}{c}1.51 \\
(0.97-2.05)\end{array}$ & $\begin{array}{c}1.67 \\
(0.99-2.14)^{\mathrm{a}} \\
\end{array}$ & 14.306 & $<0.001$ \\
\hline $\mathrm{HDL}-\mathrm{C}$, mean $\pm \mathrm{SD}[\mathrm{mmol} / \mathrm{l}]$ & $1.35 \pm 0.35$ & $1.39 \pm 0.37$ & $1.41 \pm 0.38$ & $1.48 \pm 0.36^{a}$ & 5.642 & 0.018 \\
\hline LDL-C, mean $\pm \mathrm{SD}[\mathrm{mmol} / \mathrm{l}]$ & $2.77 \pm 0.83$ & $2.95 \pm 0.81$ & $3.10 \pm 0.71^{a}$ & $3.10 \pm 0.73^{\mathrm{a}}$ & 9.005 & 0.003 \\
\hline MoCA score, mean \pm SD & $24.86 \pm 2.41$ & $23.61 \pm 3.39^{a}$ & $22.69 \pm 3.25^{a b}$ & $21.23 \pm 3.07^{a b c}$ & 68.208 & $<0.001$ \\
\hline $\mathrm{sCD} 36[\mathrm{ng} / \mathrm{ml}]^{\dagger}$ & $\begin{array}{c}2.84 \\
(2.23-3.40)\end{array}$ & $\begin{array}{c}3.38 \\
(2.58-4.18)^{\mathrm{a}}\end{array}$ & $\begin{array}{c}3.34 \\
(2.51-4.33)^{\mathrm{a}}\end{array}$ & $\begin{array}{c}3.34 \\
(2.63-4.77)^{\mathrm{a}}\end{array}$ & 30.299 & $<0.001$ \\
\hline
\end{tabular}

${ }^{a}$ Compared to $Q 1, p<0.05,{ }^{b}$ compared to $Q 2, p<0.05$, 'compared to $Q 3, p<0.05 .{ }^{\dagger} T G$ and $S C D 36$ had a skew distribution and were log transformed for statistical analyses, then back-transformed to their natural units for presentation and expressed as median (25-75\%).

A

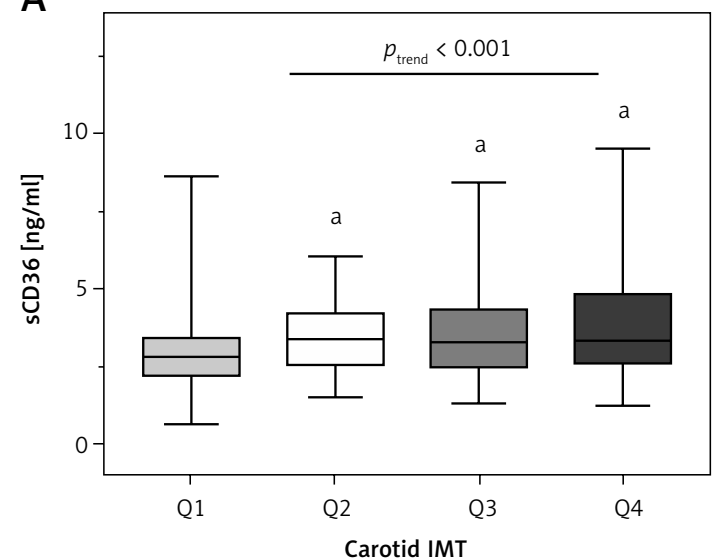

B

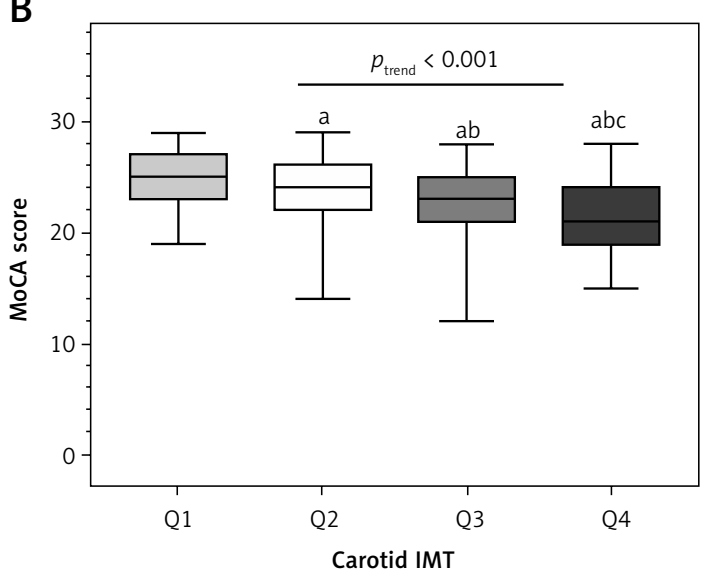

Figure 1. $\mathrm{sCD} 36(\mathrm{~A})$ and MoCA score (B) across quartiles (Q1-Q4) of carotid IMT ${ }^{a}$ Compared to $Q 1, p<0.05,{ }^{b}$ compared to $Q 2, p<0.05$, compared to $Q 3, p<0.05$. 
Table III. Characteristics of participants according to quartiles of plasma sCD36

\begin{tabular}{|c|c|c|c|c|c|c|}
\hline \multirow[t]{2}{*}{ Parameter } & \multicolumn{4}{|c|}{ sCD36 [ng/ml] } & \multirow[t]{2}{*}{$F / \chi^{2}$} & \multirow[t]{2}{*}{$P_{\text {trend }}$} \\
\hline & $\begin{array}{c}\mathrm{Q} 1 \\
(0.723-2.379) \\
(n=89)\end{array}$ & $\begin{array}{c}\mathrm{Q} 2 \\
(2.386-3.036) \\
(n=89)\end{array}$ & $\begin{array}{c}\mathrm{Q3} \\
(3.052-3.751) \\
(n=89)\end{array}$ & $\begin{array}{c}\text { Q4 } \\
(3.752-11.196) \\
(n=90)\end{array}$ & & \\
\hline Age, mean \pm SD [years] & $55.62 \pm 9.47$ & $58.90 \pm 10.13$ & $56.27 \pm 9.22$ & $58.69 \pm 8.14^{a}$ & 2.846 & 0.092 \\
\hline Men, $n(\%)$ & $27(30.3)$ & $29(32.6)$ & $29(32.6)$ & $40(44.4)^{\mathrm{a}}$ & 4.835 & 0.184 \\
\hline Education, mean \pm SD [years] & $8.75 \pm 3.32$ & $7.76 \pm 3.30^{\mathrm{a}}$ & $8.30 \pm 2.59$ & $7.89 \pm 2.92$ & 2.027 & 0.155 \\
\hline $\begin{array}{l}\text { Duration of DM, mean } \pm \text { SD } \\
\text { [years] }\end{array}$ & $6.29 \pm 3.12$ & $6.25 \pm 3.80$ & $5.19 \pm 2.89^{a b}$ & $6.37 \pm 4.17^{c}$ & 0.241 & 0.624 \\
\hline BMI, mean $\pm \mathrm{SD}\left[\mathrm{kg} / \mathrm{m}^{2}\right]$ & $25.01 \pm 3.23$ & $25.40 \pm 3.10$ & $25.48 \pm 3.57$ & $25.35 \pm 3.22$ & 0.280 & 0.756 \\
\hline $\begin{array}{l}\text { Waist circumference, } \\
\text { mean } \pm \mathrm{SD}[\mathrm{cm}]\end{array}$ & $85.13 \pm 9.39$ & $85.47 \pm 8.35$ & $86.08 \pm 9.83$ & $86.23 \pm 8.95$ & 0.020 & 0.980 \\
\hline Habitual alcohol drinker, $n$ (\%) & $10(11.2)$ & $6(6.8)$ & $10(11.2)$ & $10(11.2)$ & 1.418 & 0.701 \\
\hline Current smoker, $n(\%)$ & $15(16.9)$ & $11(12.5)$ & $14(15.7)$ & $20(22.5)$ & 3.267 & 0.352 \\
\hline Hypertension, $n$ (\%) & $43(48.3)$ & $52(58.4)$ & $42(47.2)$ & $50(55.6)$ & 3.219 & 0.359 \\
\hline $\mathrm{SBP}$, mean $\pm \mathrm{SD}[\mathrm{mm} \mathrm{Hg}]$ & $131.85 \pm 16.31$ & $133.93 \pm 16.05$ & $128.93 \pm 16.30^{b}$ & $134.14 \pm 17.25^{c}$ & 0.058 & 0.810 \\
\hline DBP, mean \pm SD $[\mathrm{mm} \mathrm{Hg}]$ & $83.76 \pm 9.75$ & $83.20 \pm 9.27$ & $82.87 \pm 8.95$ & $83.66 \pm 9.35$ & 0.023 & 0.881 \\
\hline $\mathrm{FBG}$, mean $\pm \mathrm{SD}[\mathrm{mmol} / \mathrm{l}]$ & $9.50 \pm 2.37$ & $9.46 \pm 2.39$ & $9.77 \pm 2.82$ & $10.31 \pm 2.98^{\mathrm{ab}}$ & 4.765 & 0.030 \\
\hline $\mathrm{HbA}_{1 c}$, mean $\pm \mathrm{SD}(\%)$ & $7.87 \pm 1.06$ & $7.84 \pm 1.04$ & $7.99 \pm 1.28$ & $8.00 \pm 1.27$ & 0.999 & 0.318 \\
\hline $\mathrm{TC}$, mean $\pm \mathrm{SD}[\mathrm{mmol} / \mathrm{l}]$ & $5.14 \pm 0.98$ & $5.03 \pm 1.15$ & $5.31 \pm 0.86$ & $5.26 \pm 1.07$ & 1.713 & 0.191 \\
\hline $\begin{array}{l}\text { TG, median (25-75\%) } \\
{[\mathrm{mmol} / \mathrm{l}]}\end{array}$ & $\begin{array}{c}1.22 \\
(0.90-1.90)\end{array}$ & $\begin{array}{c}1.26 \\
(0.93-1.85)\end{array}$ & $\begin{array}{c}1.28 \\
(0.94-2.06)\end{array}$ & $\begin{array}{c}1.53 \\
(0.94-2.08)\end{array}$ & 3.698 & 0.055 \\
\hline $\mathrm{HDL}-\mathrm{C}$, mean $\pm \mathrm{SD}[\mathrm{mmol} / \mathrm{l}]$ & $1.39 \pm 0.37$ & $1.42 \pm 0.40$ & $1.48 \pm 0.33$ & $1.41 \pm 0.36^{c}$ & 0.324 & 0.570 \\
\hline LDL-C, mean \pm SD $[\mathrm{mmol} / \mathrm{l}]$ & $2.86 \pm 0.74$ & $2.89 \pm 0.78$ & $3.01 \pm 0.75$ & $3.12 \pm 0.83^{\mathrm{ab}}$ & 6.193 & 0.013 \\
\hline MoCA score, mean \pm SD & $22.89 \pm 3.61$ & $23.25 \pm 3.04$ & $23.47 \pm 3.01$ & $22.81 \pm 3.51$ & $<0.001$ & 0.998 \\
\hline IMT, mean $\pm \mathrm{SD}[\mathrm{mm}]$ & $0.80 \pm 0.19$ & $0.81 \pm 0.19$ & $0.81 \pm 0.18$ & $0.90 \pm 0.16^{\mathrm{abc}}$ & 13.110 & $<0.001$ \\
\hline
\end{tabular}

${ }^{a}$ Compared to $Q 1, p<0.05,{ }^{b}$ compared to $Q 2, p<0.05$, 'compared to $Q 3, p<0.05$.

cation, FBG, TG, LDL-C, and carotid IMT $(r=0.266$, $p<0.001$ ) (Figure $3 \mathrm{~A}$ ). Correlations of LDL-C and carotid IMT with SCD36 still remained after adjustment for age, gender, and education level $(r=0.202, p<0.001)$. Carotid IMT was significantly associated with age, sex, education, duration of DM, history of hypertension, BMI, waist circumference, SBP, DBP, FBG, $\mathrm{HbA}_{1 c}$, TC, TG, LDL-C, MoCA score $(r=-0.444, p<0.001)$ (Figure $3 \mathrm{~B}$ ) and SCD36. Multivariate analysis showed that IMT was associated with $\mathrm{HbA}_{1 \mathrm{c}}$, TC, TG, LDL-C, MoCA scores $(r=0.284, p<0.001)$ and SCD36 $(r=0.200$, $p<0.001)$ after adjustment for age, gender, education level, duration of DM and history of hypertension. Results of univariate analysis showed that MoCA score was significantly correlated with age, sex, education, duration of DM, history of hypertension, BMI, waist circumference, SBP, DBP, FBG, $\mathrm{HbA}_{1 \mathrm{c}}$, LDL-C and carotid IMT. However, it was only correlated with DBP and carotid IMT after adjustment for age, gender, education level, duration of DM and history of hypertension (Table IV). No correlation between sCD36 and MoCA scores was observed in this study $(r=-0.038, p=0.470)$ (Figure $3 \mathrm{C})$.

\section{Discussion}

In this cross-sectional study, we found that soluble CD36 concentrations were associated with carotid IMT, which has a strong association with subclinical and/or silent atherosclerosis [23], and also is a strong predictor of CVD [24]. Furthermore, IMT was inversely correlated with cognitive function in diabetic patients, which was in accordance with the results of previous research $[25,26]$. However, we did not find any relationship between SCD36 and MoCA scores, indicating that concentrations of soluble sCD36 may have no correlation with cognitive function of patients with type 2 diabetes.

Previous studies have demonstrated an association between high CD36 expression and atherosclerosis risk [27], and it may become a useful tool in cardiovascular disease risk stratification [28]. 

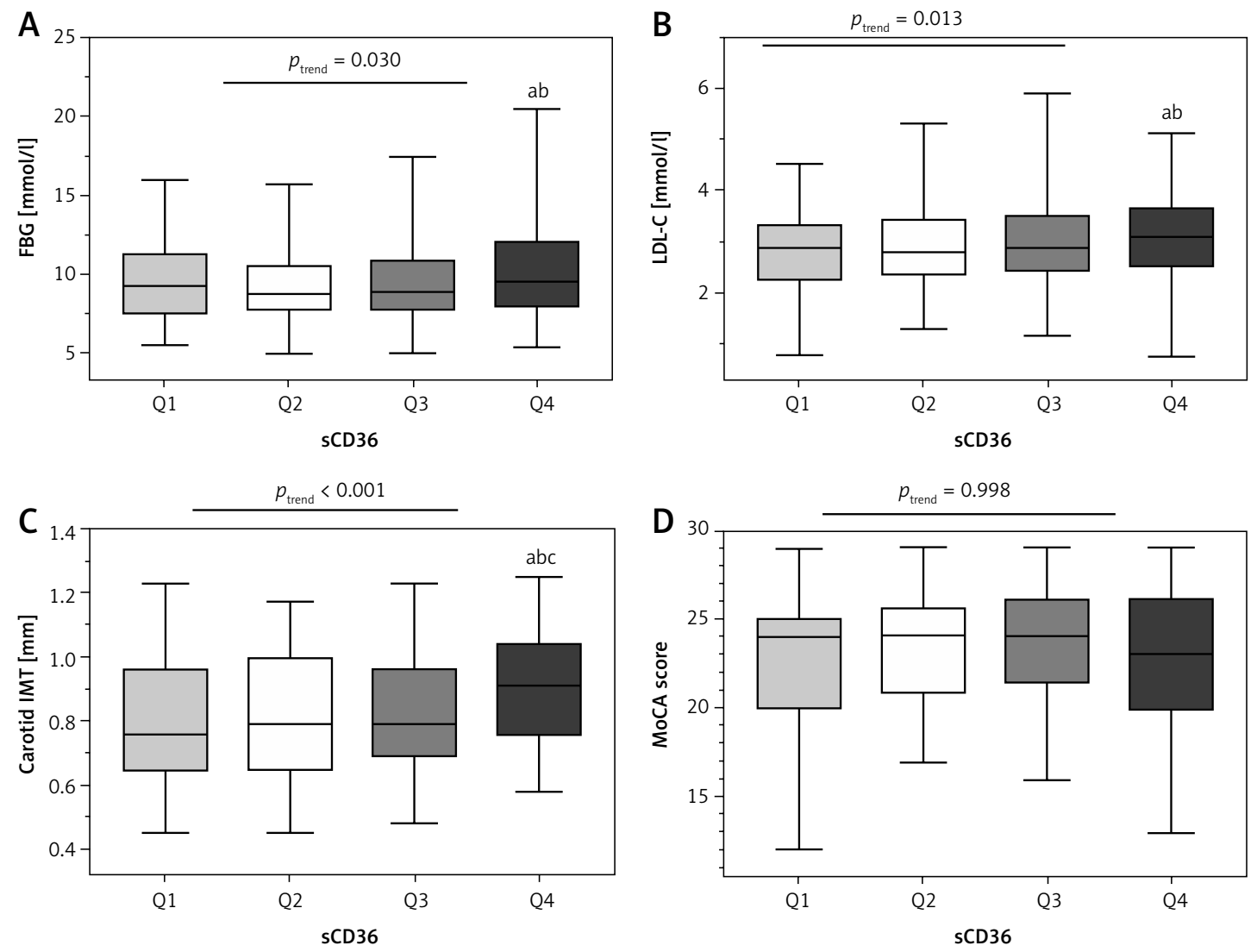

Figure 2. FBG (A), LDL-C (B), carotid IMT (C) and MoCA score (D) across quartiles (Q1-Q4) of SCD36 ${ }^{a}$ Compared to $Q 1, p<0.05$; ${ }^{b}$ compared to $Q 2, p<0.05$, compared to $Q 3, p<0.05$.
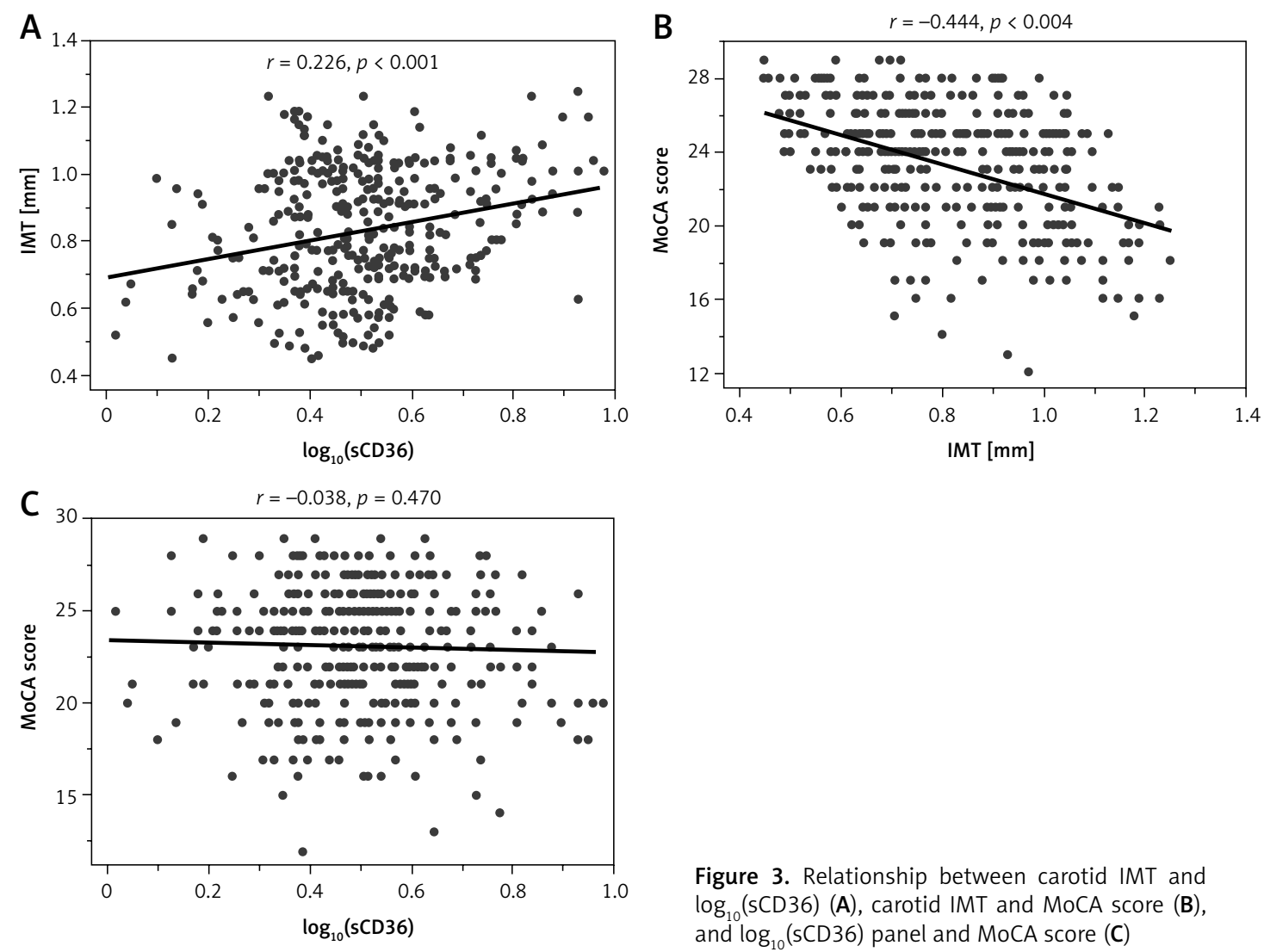

Figure 3. Relationship between carotid IMT and $\log _{10}(\mathrm{sCD} 36)(\mathrm{A})$, carotid IMT and MoCA score (B), and $\log _{10}(\mathrm{sCD} 36)$ panel and MoCA score (C) 
Table IV. Univariate and multivariate analyses of the relationships among SCD36, carotid IMT, MoCA score and other clinical characteristics

\begin{tabular}{|c|c|c|c|c|c|c|}
\hline \multirow[t]{2}{*}{ Parameter } & \multicolumn{2}{|c|}{$\mathrm{sCD} 36^{\mathrm{a}}$} & \multicolumn{2}{|c|}{ Carotid IMT } & \multicolumn{2}{|c|}{ MoCA score } \\
\hline & $\begin{array}{c}\text { Univariate } \\
\text { analysis }\end{array}$ & $\begin{array}{l}\text { Multivariate } \\
\text { analysis }{ }^{\S}\end{array}$ & $\begin{array}{l}\text { Univariate } \\
\text { analysis }\end{array}$ & $\begin{array}{l}\text { Multivariate } \\
\text { analysis }\end{array}$ & $\begin{array}{l}\text { Univariate } \\
\text { analysis }\end{array}$ & $\begin{array}{l}\text { Multivariate } \\
\text { analysis }\end{array}$ \\
\hline Age & $0.129^{*}$ & / & $0.516^{\star \star}$ & / & $-0.381^{\star *}$ & / \\
\hline Gender ${ }^{\ddagger}$ & $-0.105^{\star}$ & I & $-0.125^{\star}$ & I & -0.054 & I \\
\hline Education & $-0.133^{\star}$ & I & $-0.280^{\star \star}$ & I & $0.275^{\star \star}$ & I \\
\hline Duration of DM & 0.061 & 0.054 & $0.129^{*}$ & / & $-0.138^{\star \star}$ & / \\
\hline BMI & 0.061 & 0.008 & $0.249^{* \star}$ & 0.071 & $-0.155^{\star *}$ & -0.007 \\
\hline Waist circumference & 0.079 & -0.015 & $0.344^{* *}$ & $0.109^{*}$ & $-0.207^{\star *}$ & -0.036 \\
\hline $\begin{array}{l}\text { Habitual alcohol } \\
\text { drinker }{ }^{\ddagger}\end{array}$ & 0.014 & -0.039 & -0.029 & -0.050 & 0.057 & 0.006 \\
\hline Current smoker ${ }^{\ddagger}$ & 0.078 & 0.028 & 0.052 & -0.035 & 0.051 & 0.027 \\
\hline Hypertension ${ }^{\ddagger}$ & 0.027 & 0.036 & $0.334^{\star \star}$ & 1 & $-0.233^{* *}$ & I \\
\hline SBP & 0.045 & -0.005 & $0.258^{* *}$ & 0.024 & $-0.195^{\star *}$ & -0.048 \\
\hline DBP & 0.027 & 0.006 & $0.160^{* *}$ & 0.089 & $-0.138^{\star *}$ & $-0.109^{*}$ \\
\hline FBG & $0.138^{\star \star}$ & 0.099 & $0.141^{* \star}$ & 0.100 & $-0.112^{*}$ & -0.056 \\
\hline $\mathrm{HbA}_{1 \mathrm{c}}$ & 0.037 & 0.005 & $0.124^{*}$ & $0.105^{*}$ & $-0.123^{*}$ & -0.102 \\
\hline TC & 0.099 & 0.093 & $0.197^{*}$ & $0.129^{*}$ & -0.087 & 0.011 \\
\hline $\mathrm{TG}^{\mathrm{a}}$ & $0.108^{*}$ & 0.093 & $0.201^{\star \star}$ & $0.122^{*}$ & -0.047 & 0.009 \\
\hline HDL-C & -0.007 & -0.034 & $0.108^{*}$ & 0.005 & -0.048 & 0.074 \\
\hline LDL-C & $0.160^{\star *}$ & $0.154^{* *}$ & $0.177^{\star *}$ & $0.108^{*}$ & $-0.119^{*}$ & -0.027 \\
\hline MoCA score & -0.038 & 0.049 & $-0.444^{\star *}$ & $-0.284^{* *}$ & 1 & 1 \\
\hline sCD36a & I & I & $0.266^{* *}$ & $0.200^{* *}$ & -0.038 & 0.033 \\
\hline Carotid IMT & $0.266^{* *}$ & $0.202^{* *}$ & 1 & 1 & $-0.444^{*}$ & $-0.284^{* *}$ \\
\hline
\end{tabular}

${ }^{\S}$ After adjustment for age, gender, and education level, ' after adjustment for age, gender, education level, duration of DM, and hypertension, ${ }^{a} p$-values based on statistical analyses of log-normalized values. ${ }^{*} P<0.05,{ }^{* *} p<0.01$; ${ }^{\ddagger}$ Spearman rank correlation.

Handberg et al. [20] identified the existence and availability of soluble CD36 in plasma for further research discovery in 2008 . Recently, they suggested that both too much and too little SCD36 may be associated with an increased atherosclerosis risk in clinically healthy people [29]. Previous studies have indicated that carotid IMT is a risk marker of micro- and macrovascular complications in diabetic patients [30, 31]. Our findings of significant correlations between SCD36 and carotid IMT in type 2 diabetic patients are partly in agreement with the results of Handberg et al. [29] in a nondiabetic population. However, unlike the J-shape relationship between SCD36 and carotid IMT in the research of Handberg et al., the results of our study show lower levels of SCD36 in the lowest quartile of IMT (Q1) than upper ones (Q2-Q4) (Figure $1 \mathrm{~A})$, and higher IMT in the highest quartile of sCD36 (Q4) than lower ones (Q1-Q3) (Figure 2 C). These findings suggest that plasma sCD36 may be elevated at the early stage of atherosclerosis, but may not sensitively predict the progression of increased IMT in the diabetic population.

Biological markers are becoming important in early diagnosis of cognitive impairment. Brain-derived neurotrophic factor (BDNF) has been shown to protect neurons from damage caused by infection or injury [32]. A study of Zhen et al. indicated that decreased BDNF [33] could be associated with cognitive deficits in patients with type 2 diabetes. Qin et al. determined that the absence of CD36 could rescue the angiogenesis deficit in mice with the BDNF SNP [34]. Park et al. [19] found that Tg2576 mice lacking CD36 have a selective reduction in amyloid- $\beta 40(A \beta 1-40)$ and cerebral amyloid angiopathy (CAA), which contribute to the occurrence of $A D$, indicating that CD36 may lead to cognitive deficits in rodents. Another study [35] reported that compared with wild-type brains, CD36 knock-out mouse brains showed less accu- 
mulation of microglia, which is associated with $A \beta$ deposition. Additionally, the study of Stewart et al. [36] showed that amyloid-beta triggers inflammatory signaling through a heterodimer of toll-like receptors 4 and 6 regulated by signals from CD36. These results suggest that CD36 may play a key role in the proinflammatory events associated with $A D$ and cognitive impairment. However, it remains unknown whether increased expression of CD36 and its plasma marker SCD36 can be a risk marker of cognitive deficits in patients with diabetes. On the other hand, Giunta et al. [37] reported decreased leukocyte expression of CD36 in patients with Alzheimer's disease and mild cognitive impairment. Also, Wang et al. [38] found that AD-like pathogenesis increased oxidative stress, exacerbated inflammation, and was associated with a marked reduction in CD36 expression, and intranasal therapy with a lentiviral vector encoding human nuclear factor erythroid-2-related factor 2 (Nrf2) can diminish $A \beta$ deposition and improve spatial memory defects by directly increasing CD36 expression. Additionally, Yamanaka et al. [39] reported that activation of PPAR $\gamma$, which is mediated by upregulation of CD36 expression, may result in improved microglial A $\beta$ phagocytosis. It seems that CD36 may have a dual-directional regulation effect on the central nervous system. The expression of CD36 may not reflect the cognitive function or predict risks of cognitive impairment in diabetic patients. Future longitudinal studies investigating the interrelationship among CD36, atherosclerosis and cognitive impairment in type 2 diabetes are warranted.

Our study is limited by its cross-sectional design, and residual confounding and reverse causation are potential concerns, as in any cross-sectional study. Moreover, all the findings corresponding to carotid IMT are not necessarily coronary atherosclerosis as well as cardiovascular events. Cohort studies are necessary to determine the association of sCD36 concentrations and future cardiovascular events in patients with type 2 diabetes. The sample size in our study was relatively small, so it remains a possibility that there is a real association between SCD36 and cognitive function among diabetic patients and that our study was underpowered to detect it. Additionally, a preselected highrisk population was chosen in this study, which may considerably bias the sensitivity and specificity of the tests.

In conclusion, our study shows that a higher level of plasma SCD36 is associated with carotid IMT in patients with type 2 diabetes, independent of age, gender, education level and duration of diabetes. Also, carotid IMT is inversely correlated with cognitive function in the same subjects. However, we failed to detect a significant association between sCD36 and MoCA scores, suggesting that elevated sCD36 cannot be a risk marker of cognitive deficits in type 2 diabetic patients.

\section{Acknowledgments}

This work was supported by a joint research project (PKJ2014-Y29and HMSF-16-22-031, to CRH; PKJ2013-Y28 and PRxkq2013-01, to JXZ).

\section{Conflict of interest}

The authors declare no conflict of interest.

\section{References}

1. Brun E, Nelson RG, Bennett PH, et al. Diabetes duration and cause-specific mortality in the Verona Diabetes Study. Diabetes Care 2000; 23: 1119-23.

2. Haffner SM, Lehto S, Rönnemaa T, Pyörälä K, Laakso M. Mortality from coronary heart disease in subjects with type 2 diabetes and in nondiabetic subjects with and without prior myocardial infarction. N Engl J Med 1998; 339: 229-34.

3. Papatheodorou K, Banach M, Edmonds M, Papanas N, Papazoglou D. Complications of diabetes. J Diabetes Res 2015; 2015: 189525.

4. Zhang X, Sun Z, Jiang H, Song X. Relationship between single nucleotide polymorphisms in the 3 '-untranslated region of the vascular endothelial growth factor gene and susceptibility to diabetic peripheral neuropathy in China. Arch Med Sci 2014; 10: 1028-34.

5. Arvanitakis Z, Wilson RS, Bienias JL, Evans DA, Bennett DA. Diabetes mellitus and risk of Alzheimer disease and decline in cognitive function. Arch Neurol 2004; 61: 661-6.

6. Luchsinger JA, Reitz C, Patel B, Tang MX, Manly JJ, Mayeux R. Relation of diabetes to mild cognitive impairment. Arch Neurol 2007; 64: 570-5.

7. Mayeda ER, Haan MN, Kanaya AM, Yaffe K, Neuhaus J. Type 2 diabetes and 10-year risk of dementia and cognitive impairment among older Mexican Americans. Diabetes Care 2013; 36: 2600-6.

8. Akter K, Lanza EA, Martin SA, Myronyuk N, Rua M, Raffa RB. Diabetes mellitus and Alzheimer's disease: shared pathology and treatment? Br J Clin Pharmacol 2011; 71: 365-76.

9. Cukierman T, Gerstein HC, Williamson JD. Cognitive decline and dementia in diabetes - systematic overview of prospective observational studies. Diabetologia 2005; 48: 2460-9.

10. Chen R, Zhao X, Gu Z, et al. Serum levels of 25-hydroxyvitamin $D$ are associated with cognitive impairment in type 2 diabetic adults. Endocrine 2014; 45: 319-24.

11. Reis JP, Launer LJ, Terry JG, et al. Subclinical atherosclerotic calcification and cognitive functioning in middle-aged adults: the CARDIA study. Atherosclerosis 2013; 231: 72-7.

12. Chen RH, Jiang XZ, Zhao XH, et al. Risk factors of mild cognitive impairment in middle aged patients with type 2 diabetes: a cross-section study. Ann Endocrinol 2012; 73: 208-12.

13. Sugawara N, Yasui-Furukori N, Umeda T, et al. Comparison of ankle-brachial pressure index and pulse wave velocity as markers of cognitive function in a community-dwelling population. BMC Psychiatry 2010; 10: 46 
14. Solomon A, Kåreholt I, Ngandu T, et al. Serum cholesterol changes after midlife and late-life cognition: twentyone-year follow-up study. Neurology 2007; 68: 751-6.

15. Skoog I, Lernfelt B, Landahl S, et al. 15-year longitudinal study of blood pressure and dementia. Lancet 1996 347: 1141-5.

16. Harb D, Bujold K, Febbraio M, Sirois MG, Ong H, Marleau S. The role of the scavenger receptor CD36 in regulating mononuclear phagocyte trafficking to atherosclerotic lesions and vascular inflammation. Cardiovasc Res 2009; 83: 42-51.

17. Febbraio $M$, Silverstein RL. CD36: implications in cardiovascular disease. Int J Biochem Cell Biol 2007; 39 2012-30.

18. Nicholson AC, Han J, Febbraio M, Silversterin RL, Hajjar DP. Role of CD36, the macrophage class B scavenger receptor, in atherosclerosis. Ann N Y Acad Sci 2001; 947: 224-8.

19. Park L, Zhou J, Zhou P, et al. Innate immunity receptor CD36 promotes cerebral amyloid angiopathy. Proc Nat Acad Sci U S A 2013; 110: 3089-94.

20. Handberg A, Skjelland M, Michelsen AE, et al. Soluble CD36 in plasma is increased in patients with symptom atic atherosclerotic carotid plaques and is related to plaque instability. Stroke J Cereb Circ 2008; 39: 3092-5.

21. Handberg A, Højlund K, Gastaldelli A, et al. Plasma sCD36 is associated with markers of atherosclerosis, in sulin resistance and fatty liver in a nondiabetic healthy population. J Intern Med 2012; 271: 294-304.

22. Handberg A, Norberg M, Stenlund H, Hallmans G, Attermann J, Eriksson JW. Soluble CD36 (sCD36) clusters with markers of insulin resistance, and high sCD36 is associated with increased type 2 diabetes risk. J Clin Endocrinol Metab 2010; 95: 1939-46.

23. Lopes-Virella MF, Carter RE, Gilbert GE, et al. Risk factors related to inflammation and endothelial dysfunction in the DCCT/EDIC cohort and their relationship with nephropathy and macrovascular complications. Diabetes Care 2008; 31: 2006-12

24. Lorenz MW, Markus HS, Bots ML, Rosvall M, Sitzer M. Prediction of clinical cardiovascular events with carotid intima-media thickness: a systematic review and metaanalysis. Circulation 2007; 115: 459-67.

25. Xiang J, Zhang T, Yang QW, et al. Carotid artery atherosclerosis is correlated with cognitive impairment in an elderly urban Chinese non-stroke population. J Clin $\mathrm{Neu}$ rosci Off J Neurosurg Soc Australas 2013; 20: 1571-5.

26. Chen G, Cai L, Chen B, et al. Serum level of endogenous secretory receptor for advanced glycation end products and other factors in type 2 diabetic patients with mild cognitive impairment. Diabetes Care 2011; 34: 2586-90.

27. Silverstein RL. Inflammation, atherosclerosis, and arterial thrombosis: role of the scavenger receptor CD36. Cleve Clin J Med 2009; 76 Suppl 2: S27-30.

28. Sun Y, Scavini M, Orlando RA, et al. Increased CD36 expression signals monocyte activation among patients with type 2 diabetes. Diabetes Care 2010; 33: 2065-7.

29. Handberg A, Højlund K, Gastaldelli A, et al. Plasma sCD36 is associated with markers of atherosclerosis, in sulin resistance and fatty liver in a nondiabetic healthy population. J Intern Med 2012; 271: 294-304.

30. Rogowicz-Frontczak A, Araszkiewicz A, Pilacinski S, Zozulinska-Ziolkiewicz D, Wykretowicz A, Wierusz-Wysocka B. Carotid intima-media thickness and arterial stiffness in type 1 diabetic patients with and without microangiopathy. Arch Med Sci 2012; 8: 484-90.
31. de Kreutzenberg SV, Coracina A, Volpi A, et al. Microangiopathy is independently associated with presence, severity and composition of carotid atherosclerosis in type 2 diabetes. Nutr Metab Cardiovasc Dis 2011; 21: 286-93.

32. Patterson SL. Immune dysregulation and cognitive vulnerability in the aging brain: interactions of microglia, IL-1beta, BDNF and synaptic plasticity. Neuropharmacology 2015; 96: 11-8.

33. Zhen YF, Zhang J, Liu XY, et al. Low BDNF is associated with cognitive deficits in patients with type 2 diabetes. Psychopharmacology (Berl) 2013; 227: 93-100.

34. Qin L, Kim E, Ratan R, Lee FS, Cho S. Genetic variant of BDNF (Val66Met) polymorphism attenuates stroke-induced angiogenic responses by enhancing anti-angiogenic mediator CD36 expression. J Neurosci Off J Soc Neurosci 2011; 31: 775-83.

35. El Khoury JB, Moore KJ, Means TK, et al. CD36 mediates the innate host response to beta-amyloid. J Exp Med 2003; 197: 1657-66.

36. Stewart CR, Stuart LM, Wilkinson K, et al. CD36 ligands promote sterile inflammation through assembly of a Toll-like receptor 4 and 6 heterodimer. Nat Immunol 2010; 11: 155-61.

37. Giunta M, Rigamonti AE, Scarpini E, et al. The leukocyte expression of CD36 is low in patients with Alzheimer's disease and mild cognitive impairment. Neurobiol Aging 2007; 28: 515-8.

38. Wang CY, Wang ZY, Xie JW, et al. CD36 upregulation mediated by intranasal LV-NRF2 treatment mitigates hypoxia-induced progression of Alzheimer's-like pathogenesis. Antioxid Redox Signal 2014; 21: 2208-30.

39. Yamanaka M, Ishikawa T, Griep A, Axt D, Kummer MP, Heneka MT. PPARgamma/RXRalpha-induced and CD36mediated microglial amyloid-beta phagocytosis results in cognitive improvement in amyloid precursor protein/ presenilin 1 mice. J Neurosci Off J Soc Neurosci 2012; 32: 17321-31. 